\section{Gauß, Carl Friedrich}

R.-D. Hilgers ${ }^{1}$, N. Heussen ${ }^{1}$ und S. Stanzel ${ }^{2}$

${ }^{1}$ Institut für Medizinische Statistik, Universitätsklinikum der RWTH Aachen, Aachen, Deutschland

${ }^{2}$ DKFZ Heidelberg, Heidelberg, Deutschland

Lebensdaten Deutscher Mathematiker, geboren am 30. April 1777 in Göttingen, gestorben am 23. Februar 1855 in Braunschweig

Verdienste C. F. Gauß gilt als einer der bedeutendsten Mathematiker. Wie kaum ein anderer hat er ausgehend von praktischen Aufgabenstellungen ein Problem erfasst und dann bis zur höchsten theoretischen Abstraktion durchdacht.
Dies begründet sein Platz nicht nur in der Geschichte der Mathematik, sondern auch in der Astronomie, Geodäsie und Physik. Besondere Bedeutung für die Analyse von Messreihen haben seine wahrscheinlichkeitstheoretischen Schriften aus den Jahren 1821-1823, die sich aus der Abstraktion geodätischer Problemstellungen bei Landvermessungen unter anderen im Harz und in der Lüneburger Heide ergaben. Hierbei begründete er die Methode der kleinsten Quadrate zum Ausgleich von Beobachtungsfehlern und beschäftigte sich mit dem Problem der Ausgleichsgeraden.

\section{Literatur}

Wußing H (1989) Carl Friedrich Gauß. B.G. Teubner, Leipzig 